\title{
Serpientes de importancia médica y accidentes ofídicos en el municipio de Tezonapa, Veracruz (2003-2016)
}

Snakes of medical importance and ophidian accidents in the municipality of Tezonapa Veracruz (2003-2016).

$$
\text { Almaraz-Vidal Diego }
$$

Estudios Biológicos y Capacitaciones Comunitarias. Av. Aquiles Serdán No. 34, Jalapilla, Mpio. Rafael Delgado, Veracruz, México. C. P. 94410.

Autor para correspondencia: almarazv@ hotmail.com

Recibido: $18 / 02 / 2017$

Aceptado: 30/06/2017

\section{RESUMEN}

Se elaboró un estudio retrospectivo y descriptivo de los accidentes ofídicos ocurridos en el período de enero del 2003 a noviembre del 2016 en el municipio de Tezonapa, así como identificar las especies de serpientes de importancia médica ocurren en esta entidad administrativa. Fueron notificados 251 accidentes ofídicos y se identificaron cuatro especies de serpientes de importancia médica (dos Elápidos y dos Vipéridos). La mayor de los casos se presento en el verano-otoño (agosto a octubre), siendo el sexo masculino el más afectadas en el grupo de edad entre 25 a 44 años. Tezonapa presenta un elevado número de accidentes, debido que se trata de un municipio con predominio de población rural.

Palabras clave: Serpientes, distribución, registros, mordeduras, envenenamiento.

\begin{abstract}
A retrospective and descriptive study of ophidian accidents occurred in the period from January 2003 to November 2016 in the municipality of Tezonapa, as well as identifying the species of snakes of medical importance occurring in this administrative entity. 251 ophidian accidents were reported and four species of snakes of medical importance were identified (two Elápidos and two Vipéridos). The highest number of cases occurred in the summer-autumn (August to October), with the male being the most affected in the age group between 25 and 44 years. Tezonapa presents a high number of accidents, due to the fact that it is a municipality with predominance of rural population.
\end{abstract}

Keywords: Snakes, distribution, records, bites, poisoning.

\section{INTRODUCCÓN}

Las mordeduras por serpientes venenosas son uno de los principales problemas en la población rural en muchas partes del mundo, de las 3619 especies de serpientes que existen en el mundo solo el $19 \%$ son consideradas de importancia médica debido a que presentan un aparato 
venenoso (colmillos acanalados asociados a glándulas musculosas que secretan el veneno) (Hargreaves et al., 2014).Los compuestos del veneno causan distintos daños sistémicos sobre el tejido, en su mayoría asociados con procesos de mionecrosis y parálisis, pudiendo ocasionar la muerte y/o dejar secuelas permanentes, como la pérdida o limitación funcional de las extremidades (WHO, 2007, Warrell, 2010).

La mayoría de los accidentes ofídicos ocurren en zonas tropicales y subtropicales en donde hay una gran diversidad de serpientes debido que estos reptiles de temperatura corporal ectotérmica prefieren lugares húmedos y cálidos (Natera et al., 2005). En el Estado de Veracruz se registran aproximadamente 124 especies de serpientes de las cuales 21 especies son consideradas de importancia médica, y es considerado como uno de los cinco estados de la república mexicana con la más alta prevalencia de accidentes ofídicos, con una tasa de incidencia de 49.2 incidentes por cada 100,000 habitantes (Zúñiga-Carrasco y CaroLozano, 2013; Yañez-Arenas, 2014). En cuanto al municipio de Tezonapa, se han publicado algunos datos sobre mordeduras de serpientes (Yañez-Arenas, 2014); sin embargo, no se han realizado estudios que aporten información detallada sobre la situación de los accidentes ofídicos. El objetivo de este estudio fue generar información sobre las especies de serpientes de importancia médica que ocurren en este municipio y el perfil epidemiológico de las mordeduras de serpientes, el cual permitirá una adecuada atención médica.

\section{MATERIALES Y MÉTODOS}

El municipio de Tezonapa se ubica entre las coordenadas 8418.531 latitud y -
96.7482 longitud proyección WGS-84, a una altitud entre 60 y 1500 msnm. Está integrado por una cabecera municipal (Tezonapa), y 139 localidades rurales. Tiene una superficie de $351 \mathrm{Km}^{2}$, que representa un $0.048 \%$ del total del estado, colinda con los municipios de Zongolica y Omealca en Veracruz, con el estado de Oaxaca y Puebla. En las partes más bajas se alcanzan los $60 \mathrm{msnm}$, y en las montañas más altas los 1550 msnm.

En el Municipio se presentan tres tipos de climas, el clima Cálido húmedo con abundantes lluvias en verano semicálido húmedo con abundantes lluvias en verano y cálido húmedo con lluvias todo el año. La precipitación pluvial que va de los 2400 a $3100 \mathrm{~mm}$, con temperaturas medias anuales van de los $18{ }^{\circ} \mathrm{C}$ a los $26{ }^{\circ} \mathrm{C}$ (SEFIPLAN, 2016). La vegetación que se presenta en el municipio es la Selva Alta Perennifolia, Pastizal cultivado (INEGI, 2013). Las actividades humanas en el área han moldeado un paisaje que en la actualidad se encuentra dominado por áreas de agricultura como café cereza (Coffea arabica), caña de azúcar (Saccharum officinarum), maíz grano

(Zea mays), Palma camedor (Chamaedorea spp), hoja de plátano (Velillo), hule (Hevea brasiliensis), maderables como cedro rojo (Cedrela odorata) y cultivos frutales como limón persa (Citrus latifolia) entre otros (SEFIPLAN, 2016).

La información sobre las especies de importancia médica que se distribuyen en el municipio de Tezonapa se obtuvo de la consulta de la colección herpetológica de la Facultad de Ciencias Biológicas y Agropecuarias de la Universidad Veracruzana campus Peñuela, así como las bases de datos de VertNet

(http://www.vertnet.org) y Global Biodiversity Information Facility (GBIF) 
(http://www.gbif.org), y de la revisión de literatura. Los registros de mordeduras de serpientes fueron obtenidos del Sistema Único Automatizado de Vigilancia Epidemiológica de la Secretaría de Salud del periodo de enero del 2003 a noviembre del 2016. Se calculo la tasa de incidencia para el municipio (suma de los casos dividida por la población total de Veracruz por cada 100,000 habitantes), utilizando la información del Instituto Nacional de Estadística, Geografía e Informática (INEGI, 2016).

\section{RESULTADOS}

El municipio de Tezonapa se registran cuatro especies de serpientes de importancia médica: dos Vipéridos (Atropoides nummifer, Bothrops asper), y dos Elápidos (Micrurus diastema, Micrurus elegans). Esta riqueza es el resultado de una gran heterogeneidad de hábitats óptimos que presenta el municipio para las serpientes. Sin embargo los registros bibliográficos obtenidos muestran que existen grandes extensiones de territorio sin realizar investigaciones, en el cual existe la posibilidad de que concurran especies que se desconoce su presencia (Figura 1).
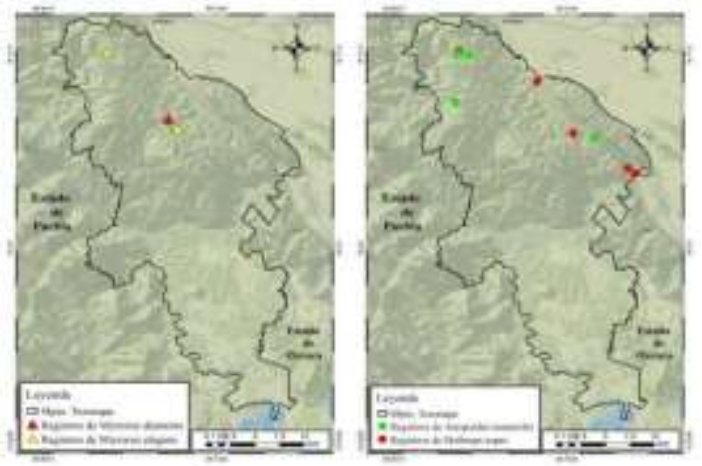

Figura 1. Registros de las especies de importancia médica (Elápidos y
Viperidos) en el municipio de Tezonapa, Veracruz.

En el periodo de enero del 2003 a noviembre del 2016 se registraron 251 accidentes ofídicos, con una tasa de incidencia de 3.09 accidentes por cada 100,000 habitantes. El mayor número de casos fue registrado en el año 2013 con 35 casos, seguido de los años 2011 y 2012 con 33 casos cada uno (Figura 2). El incremento de los accidentes en el 2013 podría deberse al aumento de las actividades económicas en la región (aumento de plantaciones de caña de azúcar y café) (eluniversalveracruz, 2014), propiciando un mayor número de trabajadores en campo.

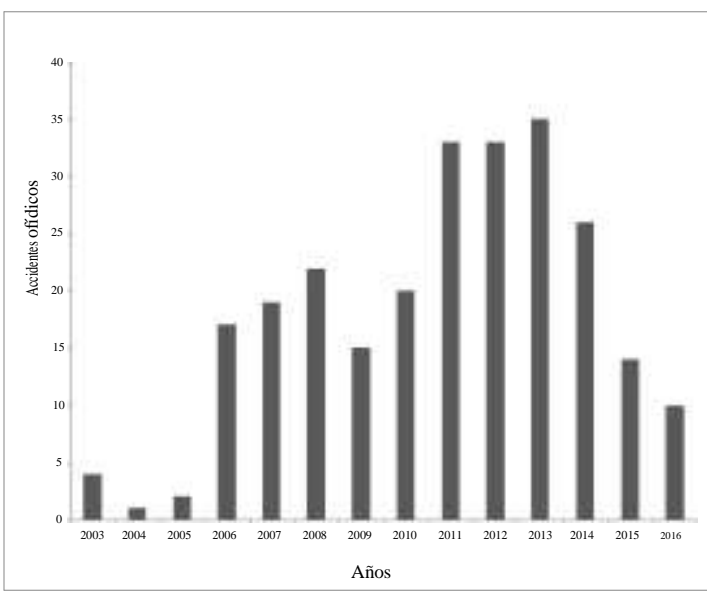

Figura 2. Distribución de los accidentes ofídicos en el municipio de Tezonapa durante el período 2003-2016.

La mayor incidencia de casos se presento en los meses de agosto a octubre con 102 casos, meses que corresponden al período de lluvias (figura 3). Algunos estudios han revelado una clara relación entre la precipitación pluvial mensual y la frecuencia de accidentes ofídicos, ya que en esta temporada se propicia el desbordamiento de ríos y arroyos, 
haciendo que las serpientes se vean obligadas a salir de sus madrigueras en busca de los lugares más secos (Bernarde y Abe, 2006); además, de que en esta temporada es cuando los nacimientos de las serpientes ocurren con mayor intensidad $\mathrm{y}$ hay aumento en la disponibilidad de presas (Natera et al., 2005).

Asimismo en estos meses de lluvias se incrementan las actividades agropecuarias en la región, existiendo una mayor exposición de riesgo de mordedura por serpiente.

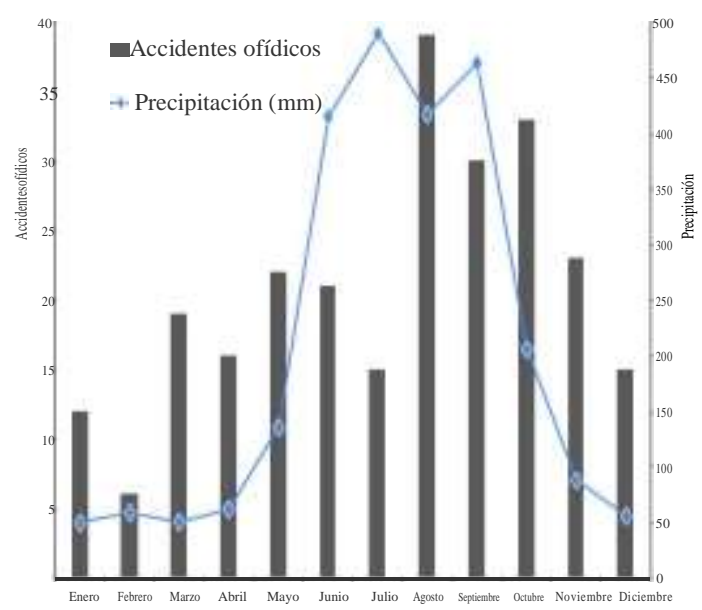

Figura 3. Distribución mensual de los accidentes de accidentes ofídicos registrados en el municipio de Tezonapa (2003-2016).

La mayoría de los casos ocurrieron en el sexo masculino 162 casos $(65 \%)$ mientras que en el femenino fue de 89 casos (35\%), por lo tanto la probabilidad de sufrir un accidente ofídico es de 1.8 veces mayor para los hombres que para las mujeres. Con relación a la distribución de los accidentes por grupo de edades, se observa que los más afectados son los comprendidos entre el rango de edad 25 a 44 años, seguido de 15 a 19 años y de los 50 a 59 años (Figura 4). Estos resultados coinciden con lo reportado por GuzmánGuzmán et al., (1993) y Luna-Bauza et al. (2004), ya que en este género y rango de edad se concentra la mayor fuerza de trabajo en el campo en labores de siembra y cosecha de los cultivos. La alta incidencia de mordeduras de serpientes en adolescentes de 15 a 19 años, puede deberse a que este grupo de edad participan en labores agrícolas y pueden ser menos precavidos (Van den Berge et al., 2008). Se resalta un número significativo en el grupo de edad de 50 a 59 años, probablemente a que existe un importante número de personas en estas edades entre los trabajadores rurales.

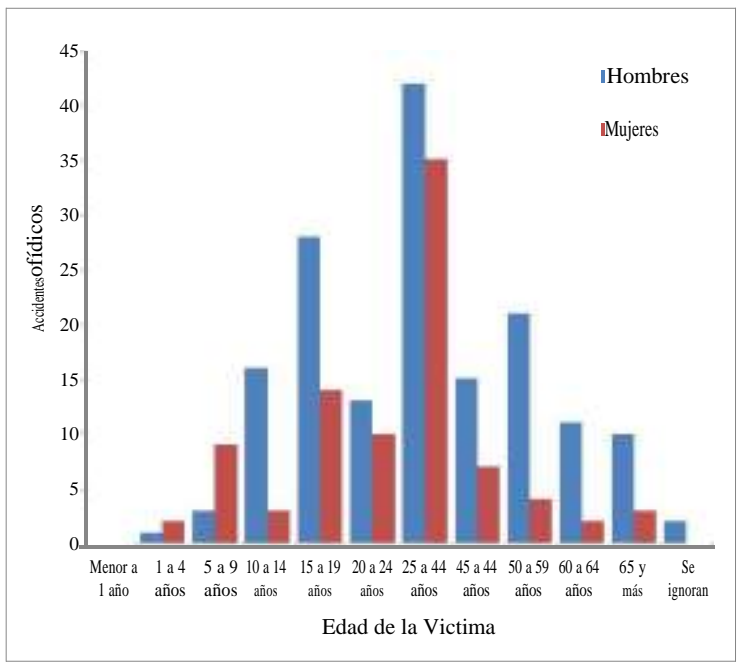

Figura 4. Distribución de los accidentes ofídicos por clase de edad y sexo ocurridos en el Mpio. De Tezonapa (2003-2016).

Con relación a las especies que pueden ocasionar los accidentes ofídicos en este municipio, no es posible identificar a cuál de las especies enlistadas se encuentra implicada, ya que estos datos no se 
registran en las estadísticas recabadas por el Sistema Único Automatizado de Vigilancia Epidemiológica. De acuerdo con Campbell y Lamar (2004) y Gutiérrez (2011), Bothrops asper puede ser la serpiente que sea la responsable en la mayoría de los accidentes debido a su amplia distribución geográfica. Esta especie tiene la capacidad de adaptarse a zonas perturbadas como pastizales y cultivos agrícolas, su coloración críptica parecida a troncos, hojarasca, y su comportamiento es impredecible, es considerada como la más peligrosa de América Latina (Sasa y Vazquez, 2003; Gutiérrez, 2011). Además de la toxicidad de su veneno (de tipo hemolítico y neurotóxico), pueden llegar a medir hasta $2.4 \mathrm{~m}$ de longitud lo que les permite inocular grandes cantidades de veneno, produciendo daños severos a la víctima y posiblemente la muerte si no recibe atención médica a tiempo (Köhler, 2003; Pérez-Higareda et al., 2007).

\section{CONCLUSIONES}

El accidente ofídico en el municipio de Tezonapa está relacionado con la actividad agrícola, aumentando su incidencia en las épocas lluviosas. El conocer la distribución geográfica de las serpientes de interés médico, así como la distribución de los accidentes ofídicos servirá para identificar zonas de mayor riesgo ofídico, y una adecuada disponibilidad de los antídotos en los diferentes lugares oportunos de la región. $\mathrm{Al}$ igual de suma importancia el capacitar activamente a las comunidades rurales en educación ambiental y la prevención del accidente ofídico. Las serpientes regulan las poblaciones naturales de sus presas al ser depredadoras importantes que mantienen la diversidad biológica, por lo cual debemos respetarlas y conservarlas.

\section{AGRADECIMIENTOS}

A la Dirección General de Epidemiología de la Secretaria de Salud y al Sistema Único Automatizado para la Vigilancia Epidemiológica por la facilitación de la base datos de las mordeduras de serpientes.

\section{LITERATURA CITADA}

Bernarde P.S. y Abe A.S. 2006. A snake community at Espigão do Oeste, Rondônia, Southwestern Amazon, Brazil. South American Journal of Herpetology (1), p. 102-113. https://doi.org/10.2994/1808-9798(2006)1

Campbell J.A. y Lamar W.W. 2004. The Venomous Reptiles of the Western Hemisphere. Cornell University Press, Vol. I-II, Ithaca, N.Y. 774 pp.

Eluniversalveracruz. 2014. Veracruz registra aumento en actividades económicas.

http://www.eluniversalveracruz.com.mx/n egocios-economia/2014/veracruz-registraaumento-en-actividades-economicas19885.html. Consultada mayo 2017.

Gutiérrez J.M. 2011. Envenenamientos por mordeduras de serpientes en América Latina y el Caribe: Una visión integral de carácter regional. Boletín de Malariología y Salud Ambiental, Vol. LI, No 1. p. 1-16.

Guzmán-Guzmán S.O., Gómez-García A., Rodríguez-García J. y Luna-Morales N. 1993. Mordeduras de serpientes venenosas en Veracruz. La Ciencia y el Hombre, julio-diciembre No. 15, p. 129144. 
Hargreaves A.D., Swain M.T., Hegarty M.J., Logan D.W. y Mulley J.F. 2014.

Restriction and recruitment-gene duplication and the origin and evolution of snake venom toxins. Genome Biological Evolution 2014; 6(8), p.20882095.

https://doi.org/10.1093/gbe/evu166

INEGI. 2013. Conjunto de datos vectoriales de Uso del Suelo y VegetaciónEscala 1:250 000, Serie V

(Capa Unión). from

http://www.inegi.org.mx/geo/conten

idos/recnat/usosuelo/Default.aspx.

Consultada diciembre 2016

INEGI. 2016. Censos y conteo de población $\quad \mathrm{y}$ vivienda. http://www.inegi.org.mx/est/contenidos/P royectos/ccpv/. Consultada diciembre 2016.

Köhler G. 2003. Reptiles of Central America. Offenbach, Alemania: Herpeton

Luna-Bauza M.E., Martínez-Ponce G. y Salazar-Hernández A.C. 2004. Mordeduras por serpiente. Panorama epidemiológico de la zona de Córdoba, Veracruz. Revista de la Facultad de

Medicina, Universidad Nacional Autónoma de México, (47), pp. 149-53.

Natera M., Almeida F. y Pérez E. 2005. Reportes recientes de accidentes ofídicos en la región noroccidental del estado Guárico, Venezuela. Herpetotropicos 2(1), p. 43-46.

Melgarejo A.R. 2003. Serpentes Peçonhentas do Brasil. En: Cardoso J.L.C., França, F.O.S. Fan, H.W. Málaque, C.M. y Haddad Jr. V. (eds).
Animais peçonhentos no brasil. Biologia, Clinica e terapêutica dos acidentes. Sarvier, São Paulo, 2003. P. 33-60.

Pérez-Higareda, G., López-Luna M.A. y Smith H.M. 2007. Serpiente de la región de Los Tuxtlas, Veracruz, México. Guía de Identificación Ilustrada. Universidad Nacional Autónoma de México. 189 pp.

Sasa, M. y Vázquez. S. 2003. Snakebite envenomation in Costa Rica: a revision of incidence in the decade 1990-2000. Toxicon. 41, p.19-22. https://doi.org/10.1016/S0041-0101(02)00

SEFIPLAN. 2016. Cuadernillos Municipales, Tezonapa. http://ceieg.veracruz.gob.mx/wpcontent/uploads/sites/21/2016/05/Tezona pa.pdf. Consultada mayo 2017.

Van den Berge, M., Baas L., Ensing A. y Quiroz L. 2008. Las peores formas de trabajo infantil en América Latina: Identificación y opciones estratégicas. Ámsterdam: Fundación IREWOC. 96 pp.

Walteros D. y Paredes A. 2014. Protocolo de Vigilancia en Salud Pública: Accidente Ofídico. Ciudad: Ministerios de Salud y Protección Social-Instituto Nacional de Salud, Grupo de Enfermedades Transmisibles Equipo de Zoonosis. 28 pp.

Warrell D.A. 2010. Snake bite. The Lancet, 375, p. 77-88. https://doi.org/10.1016/S0140-6736(09)61 
Warrel D.A. 2005. Guidelines for the Clinical Management of Snake Bite in the South-East Asia Region. World Health Organization. $162 \mathrm{pp}$.

WHO. 2007. Rabies and envenomings. A neglected public health issue. World Health Organization. Report of a consultative meeting Geneva. 38 pp.

Yañez-Arenas C. 2014. Análisis temporal y geográfico del envenenamiento por mordedura de serpiente en Veracruz, México (2003-2012). Gaceta Médica de México 150 Suppl, p. 60-4.

Zúñiga-Carrasco I.R. y Caro-Lozano J. 2013. Aspectos clínicos y epidemiológicos de la mordedura de serpientes en México. Evidencia Médica e Investigación en Salud 6(4), p. 125-136. 


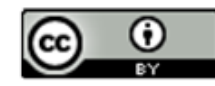

Este tex to está protegido por una licencia licencia Creative Commons 4.0 .

Usted es libre para Compartir —copiar y redistribuir el material en cualquier medio o form ato-y Adaptar el documento —remezclar, transformar y crear a partir del material- para cualquier propósito,, incluso para fines comerciales, siempre que cumpla la condición de:

Atribución: Usted debe dar crédito a la obra original de manera adecuada, proporcionar un enlace a la licencia, e indicar si se han realizado cambios. Puede hacerlo en cualquier form a razonable, pero no de form a tal que sugiera que tiene el apoyo del licenciante o lo recibe por el uso que hace de la obra.

Resumenclelicencia - Textocompletocislalicencia 